\title{
Front Matter: Volume 8374
}

, "Front Matter: Volume 8374," Proc. SPIE 8374, Next-Generation Spectroscopic Technologies V, 837401 (13 June 2012); doi: 10.1117/12.979220

Event: SPIE Defense, Security, and Sensing, 2012, Baltimore, Maryland, SPIE. United States 


\title{
PROCEEDINGS OF SPIE
}

\section{Next-Generation Spectroscopic Technologies $V$}

\author{
Mark A. Druy \\ Richard A. Crocombe \\ Editors
}

23-24 April 2012

Baltimore, Maryland, United States

Sponsored and Published by

SPIE

\section{Volume 8374}


The papers included in this volume were part of the technical conference cited on the cover and title page. Papers were selected and subject to review by the editors and conference program committee. Some conference presentations may not be available for publication. The papers published in these proceedings reflect the work and thoughts of the authors and are published herein as submitted. The publisher is not responsible for the validity of the information or for any outcomes resulting from reliance thereon.

Please use the following format to cite material from this book:

Author(s), "Title of Paper," in Next-Generation Spectroscopic Technologies V, edited by

Mark A. Druy, Richard A. Crocombe, Proceedings of SPIE Vol. 8374 (SPIE, Bellingham, WA, 2012) Article CID Number.

ISSN 0277-786X

ISBN 9780819490520

Published by

SPIE

P.O. Box 10, Bellingham, Washington 98227-0010 USA

Telephone +1 3606763290 (Pacific Time) · Fax +1 3606471445

SPIE.org

Copyright (C) 2012, Society of Photo-Optical Instrumentation Engineers

Copying of material in this book for internal or personal use, or for the internal or personal use of specific clients, beyond the fair use provisions granted by the U.S. Copyright Law is authorized by SPIE subject to payment of copying fees. The Transactional Reporting Service base fee for this volume is $\$ 18.00$ per article (or portion thereof), which should be paid directly to the Copyright Clearance Center (CCC), 222 Rosewood Drive, Danvers, MA 01923. Payment may also be made electronically through CCC Online at copyright.com. Other copying for republication, resale, advertising or promotion, or any form of systematic or multiple reproduction of any material in this book is prohibited except with permission in writing from the publisher. The CCC fee code is 0277-786X/12/\$18.00.

Printed in the United States of America.

Publication of record for individual papers is online in the SPIE Digital Library.

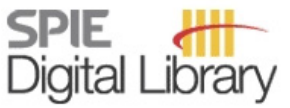

SPIEDigitalLibrary.org

Paper Numbering: Proceedings of SPIE follow an e-First publication model, with papers published first online and then in print and on CD-ROM. Papers are published as they are submitted and meet publication criteria. A unique, consistent, permanent citation identifier (CID) number is assigned to each article at the time of the first publication. Utilization of CIDs allows articles to be fully citable as soon as they are published online, and connects the same identifier to all online, print, and electronic versions of the publication. SPIE uses a six-digit CID article numbering system in which:

- The first four digits correspond to the SPIE volume number.

- The last two digits indicate publication order within the volume using a Base 36 numbering system employing both numerals and letters. These two-number sets start with 00, 01, 02, 03, 04, $05,06,07,08,09,0 A, 0 B \ldots 0 Z$, followed by 10-1Z, 20-2Z, etc.

The CID number appears on each page of the manuscript. The complete citation is used on the first page, and an abbreviated version on subsequent pages. Numbers in the index correspond to the last two digits of the six-digit CID number. 


\section{Contents}

vii Conference Committee
ix Introduction

SESSION 1 SPECTROMETERS IN THE FIELD

837402 Progress in fieldable laser-induced breakdown spectroscopy (LIBS) [8374-01]

A. W. Miziolek, U.S. Army Research Lab. (United States)

837403 Field portable time resolved SORS sensor for the identification of concealed hazards [8374-02]

B. Cletus, W. Olds, E. L. Izake, S. Sundarajoo, P. M. Fredericks, E. Jaatinen, Queensland Univ. of Technology (Australia)

837404 Miniature near-infrared (NIR) spectrometer engine for handheld applications [8374-03] N. A. O'Brien, C. A. Hulse, D. M. Friedrich, F. J. Van Milligen, M. K. von Gunten, JDSU (United States); F. Pfeifer, H. W. Siesler, Univ. of Duisburg-Essen (Germany)

837406 Advances in handheld FT-IR instrumentation [8374-05]

J. Arnó, L. Cardillo, K. Judge, M. Frayer, M. Frunzi, P. Hetherington, D. Levy, K. Oberndorfer, W. Perec, T. Sauer, J. Stein, E. Zuidema, Smiths Detection (United States)

\section{SESSION 2 ENABLING TECHNOLOGIES}

837407 Investigation of optically injected charge carrier dynamics in silicon wafers using terahertz spectroscopic imaging [8374-06]

T. Arnold, M. De Biasio, W. Muehleisen, R. Leitner, CTR Carinthian Tech Research AG (Austria)

837408 Time-resolved absolute spectral analysis of IR countermeasure flares and its experimental validation by using an optical emission spectrometer with PbSe array detector [8374-08]

H. Lee, C. Oh, J. W. Hahn, Yonsei Univ. (Korea, Republic of)

\section{SESSION 3 IMAGING AND CHEMOMETRICS I}

837409 Multi- and hyperspectral UAV imaging system for forest and agriculture applications [8374-09]

J. Mäkynen, H. Saari, C. Holmlund, R. Mannila, T. Antila, VTT Technical Research Ctr. of

Finland (Finland)

8374 OA Simple XRD algorithm for direct determination of cotton crystallinity [8374-10]

Y. Liu, USDA Agricultural Research Service(United States); D. Thibodeaux, Consultant (United

States); G. Gamble, P. Baver, USDA Agricultural Research Service (United States);

D. VanDerveer, Clemson Univ. (United States) 
$8374 \mathrm{OB} \quad$ A small, low-cost, hyperspectral imaging FTIR sensor design for standoff detection applications [8374-11]

T. Gruber, Jr., B. Moore, B. Tercha, R. Bowe, MESH, Inc. (United States)

8374 OC Video-rate chemical identification and visualization with snapshot hyperspectral imaging [8374-12]

A. Bodkin, Bodkin Design \& Engineering, LLC (United States); A. Sheinis, Univ. of

Wisconsin-Madison (United States); A. Norton, Norton Engineered Optics (United States);

J. Daly, C. Roberts, Bodkin Design \& Engineering, LLC (United States); S. Beaven,

J. Weinheimer, Space Computer Corp. (United States)

8374 OD A regularized iterative reconstruction algorithm for x-ray diffraction tomography [8374-13]

K. Chen, D. A. Castañón, Boston Univ. (United States)

\section{SESSION 4 IMAGING AND CHEMOMETRICS II}

$8374 \mathrm{OE} \quad$ Thermal hyperspectral chemical imaging [8374-14]

H. Holma, T. Hyvärinen, A.-J. Mattila, I. Kormano, Specim, Spectral Imaging Ltd. (Finland)

8374 OF Spectral imaging device based on a tuneable MEMS Fabry-Perot interferometer [8374-15] J. Antila, R. Mannila, U. Kantojärvi, C. Holmlund, A. Rissanen, I. Näkki, J. Ollila, H. Saari, VTT Technical Research Ctr. of Finland (Finland)

8374 OG Narrow band SWIR hyperspectral imaging: a new approach based on volume Bragg grating [8374-16]

M. Verhaegen, S. Lessard, S. Blais-Ovellette, Photon etc. (Canada)

SESSION 5 LASER-BASED AND CAVITY RINGDOWN SPECTROSCOPY

$8374 \mathrm{OH} \quad$ Mid-IR interband cascade lasers operating with $<\mathbf{3 0} \mathbf{m W}$ of input power [8374-17]

W. W. Bewley, C. D. Merritt, C. S. Kim, U.S. Naval Research Lab. (United States); M. Kim, Sotera Defense Solutions, Inc. (United States); C. L. Canedy, I. Vurgaftman, J. Abell, J. R. Meyer, U.S. Naval Research Lab. (United States)

83740 Ol Monolithic integrated-optic TDLAS sensors [8374-18]

M. B. Frish, D. R. Scherer, R. T. Wainner, M. G. Allen, Physical Sciences Inc. (United States);

R. Shankar, M. Lončar, Harvard Univ. (United States)

8374 OK Monolithic widely tunable quantum cascade laser [8374-20]

K. M. Lascola, R. P. Leavitt, J. D. Bruno, J. L. Bradshaw, J. T. Pham, F. J. Towner, Maxion Technologies, Inc. (United States)

$8374 \mathrm{OL}$ High-performance interband cascade lasers emitting between $\mathbf{3 . 3}$ and $\mathbf{3 . 5}$ microns [8374-21]

J. D. Bruno, R. P. Leavitt, J. L. Bradshaw, K. M. Lascola, J. T. Pham, F. J. Towner, Maxion Technologies, Inc. (United States) 
8374 OM Detection and quantification of explosives and CWAs using a handheld widely tunable quantum cascade laser [8374-22]

E. R. Deutsch, F. G. Haibach, A. Mazurenko, Block MEMS (United States)

\section{SESSION $6 \quad$ RAMAN, SERS, AND SECURITY APPLICATIONS}

8374 ON A time-resolved 128x128 SPAD camera for laser Raman spectroscopy [8374-23]

Y. Maruyama, Delft Univ. of Technology (Netherlands); J. Blacksberg, Jet Propulsion Lab.

(United States); E. Charbon, Delft Univ. of Technology (Netherlands)

837400 Identification of targets at remote distances with Raman spectroscopy [8374-24]

R. Cox, B. Williams, M. H. Harpster, DeltaNu, Inc. (United States)

8374 OP Fiber-optic Raman probe based on single-crystal sapphire fiber [8374-25]

T. Liu, M. Han, C. Raml, D. R. Alexander, X. He, Y. Lu, Univ. of Nebraska-Lincoln (United States)

$83740 Q \quad$ Portable Raman spectroscopy using retina-safe (1550 $\mathbf{~ m}$ ) laser excitation [8374-26]

C. Brouillette, W. Smith, M. Donahue, H. Huang, C. Shende, A. Sengupta, F. Inscore, M. Patient, S. Farquharson, Real-Time Analyzers, Inc. (United States)

8374 OS Industrial Raman mapping spectroscopy for mining applications [8374-28]

M. De Biasio, T. Arnold, G. McGunnigle, R. Leitner, A. Tortschanoff, Carinthian Tech Research AG (Austria); N. Fietz, L. Weitkämper, RWTH Aachen (Germany); D. Balthasar, V. Rehrmann, TOMRA Sorting GmbH (Germany)

SESSION 7 NOVEL SPECTROMETERS I

8374 OT Pulsed and high-speed FTIR spectroscopy [8374-36]

S. P. Heussler, National Univ. of Singapore (Singapore); H. O. Moser, Karlsruhe Institute of Technology (Germany); S. M. P. Kalaiselvi, C. Quan, C. J. Tay, S. P. Turaga, M. Breese, National Univ. of Singapore (Singapore)

8374 OU High-speed resonant FTIR spectrometer [8374-30]

J. Rentz Dupuis, D. Carlson, D. J. Mansur, S. P. Newbry, R. Vaillancourt, J. R. Engel, OPTRA, Inc. (United States); B. Engel, Nelson Air Corp. (United States)

8374 OV A new high-resolution, high-throughput spectrometer: first experience as applied to Raman spectroscopy [8374-31]

J. T. Meade, Arjae Spectral (Canada); B. B. Behr, Arjae Spectral (United States); A. R. Hajian, Tornado Medical Systems (Canada)

\section{SESSION $8 \quad$ NOVEL SPECTROMETERS II}

8374 OW Realization of a hybrid-integrated MEMS scanning grating spectrometer [8374-32]

T. Pügner, J. Knobbe, H. Grüger, H. Schenk, Fraunhofer Institute for Photonic Microsystems (Germany) 
8374 OX Widely tunable Fabry-Perot filter based MWIR and LWIR microspectrometers [8374-33] M. Ebermann, N. Neumann, InfraTec GmbH (Germany); K. Hiller, Chemnitz Univ. of Technology (Germany); E. Gittler, Jenoptik Optical Systems GmbH (Germany); M. Meinig, S. Kurth, Fraunhofer ENAS (Germany)

8374 OY A compact optical spectrometer based on a single-grating Fresnel diffractive optical element [8374-34]

C. Yang, P. S. Edwards, K. Shi, Z. Liu, The Pennsylvania State Univ. (United States)

$8374 \mathrm{OZ}$ Compact low-cost waveguide-based optical spectrometer for detection of chemical/biological agents [8374-35]

B. Bergner, Spectrum Scientific, Inc. (United States); P. Kumar, Wayne State Univ. (United States); D. Cook, Spectrum Scientific, Inc (United States); I. Avrutsky, Wayne State Univ. (United States)

$837410 \quad$ Fourier transform infrared phase shift cavity ring down spectrometer [8374-29]

J. Rentz Dupuis, J. R. Engel, OPTRA, Inc. (United States)

$837411 \quad$ Chemical imaging using infrared photothermal microspectroscopy [8374-37] R. Furstenberg, C. A. Kendziora, M. R. Papantonakis, V. Nguyen, R. A. McGill, U.S. Naval Research Lab. (United States)

\section{POSTER SESSION}

837413 Snapshot spectral imaging using optimized diffractive optical elements [8374-39] M. De Biasio, T. Arnold, A. Tortschanoff, CTR Carinthian Tech Research AG (Austria); A. Hermerschmidt, HOLOEYE Photonics AG (Germany); R. Leitner, CTR Carinthian Tech Research AG (Austria)

$837414 \quad \mathrm{NO}$ and $\mathrm{N}_{2} \mathrm{O}$ detection employing cavity-enhanced technique [8374-40] J. Wojtas, R. Medrzycki, B. Rutecka, J. Mikolajczyk, M. Nowakowski, D. Szabra, M. Gutowska, Military Univ. of Technology (Poland); T. Stacewicz, Univ. of Warsaw (Poland); Z. Bielecki, Military Univ. of Technology (Poland)

Author Index 


\title{
Conference Committee
}

\author{
Symposium Chair \\ Kevin P. Meiners, Office of the Secretary of Defense (United States) \\ Symposium Cochair
}

Kenneth R. Israel, Lockheed Martin Corporation (United States)

Conference Chairs

Mark A. Druy, Physical Sciences Inc. (United States)

Richard A. Crocombe, Thermo Fisher Scientific Inc. (United States)

Program Committee

John Marcell Dell, The University of Western Australia (Australia)

Richard D. Driver, Headwall Photonics Inc. (United States)

Michael B. Frish, Physical Sciences Inc. (United States)

David M. Haaland, Spectral Resolutions (United States)

Frederick G. Haibach, Block Engineering, LLC (United States)

Martin Kraft, Carinthian Tech Research AG (Austria)

Jouko O. Malinen, VTT Technical Research Centre of Finland (Finland)

Curtis A. Marcott, Light Light Solutions, LLC (United States)

Ellen V. Miseo, Analytical Answers, Inc. (United States)

David W. Schiering, Smiths Detection (United States)

Eric B. Takeuchi, Daylight Solutions Inc. (United States)

Christopher J. White, Active Spectrum, Inc. (United States)

Session Chairs

1 Spectrometers in the Field

Mark A. Druy, Physical Sciences Inc. (United States)

2 Enabling Technologies

Michael Radunsky, Daylight Solutions Inc. (United States)

3 Imaging and Chemometrics I

Richard A. Crocombe, Thermo Fisher Scientific Inc. (United States)

$4 \quad$ Imaging and Chemometrics II

Richard A. Crocombe, Thermo Fisher Scientific Inc. (United States) 
$5 \quad$ Laser-based and Cavity Ringdown Spectroscopy

Michael B. Frish, Physical Sciences Inc. (United States)

6 Raman, SERS, and Security Applications

David W. Schiering, Smiths Detection (United States)

$7 \quad$ Novel Spectrometers I

Richard A. Crocombe, Thermo Fisher Scientific Inc. (United States)

8 Novel Spectrometers II

Frederick G. Haibach, Block Engineering, LLC (United States) 


\section{Introduction}

The past twenty years have seen a massive investment in photonics, electronics and MEMS, aimed at developing new telecommunications capabilities and innovative consumer products. These investments have led to advances in miniature optics, light sources, tunable filters, array detectors, fiber optic sensors, and a range of other photonic devices, across the whole electromagnetic spectrum, along with technologies for their mass production. In addition, there have been remarkable developments in handheld consumer electronics (cell phones, RF technology, processors, operating systems, displays, user interfaces, memory, Bluetooth, WiFi, cameras, accelerometers, GPS, etc.). All of these advances are increasingly being exploited in new spectroscopic instruments, and are now poised to be the basis of next generation handheld scientific instruments.

Portable and handheld instruments are being developed that are often more sensitive and selective, smaller, cheaper, and more robust than their laboratory predecessors. Concurrent improvements in analytical theory, data analysis methods and portable processors enable these spectroscopic devices to give specific, actionable, answers to their non-specialist operators. Spectroscopybased systems are now making critical judgments in environments and applications that were unreachable twenty years ago, from hazardous materials to the operating theater, and from field geologists to customs and border personnel.

Advances in array detectors (CCD, CID, InGaAs, InSb, MCT, CMOS, etc.) are enabling a new generation of faster imaging spectrometers, with both laboratory and field applications. Lower-cost infrared arrays have been developed, employing MEMS techniques. Again, advances in laser sources, particularly the mid-infrared are for the first time being used in combination with advances in detector technology to create new spectroscopic platforms.

The emphasis in this conference is on advanced technologies for spectroscopic instrumentation, particularly the infrared, near-infrared, and Raman molecular techniques, but also including advances enabling miniature and portable spectrometers across the electromagnetic spectrum, including $x$-ray fluorescence, teraHertz, electron spin resonance, nuclear magnetic resonance and mass spectrometry.

This conference premiered at Optics East 2007 in Boston, MA and is now part of the Defense Sensing \& Security Symposium. In 2012, the conference spanned two days, and was divided into sessions focusing on: Spectrometers in the Field; Enabling Technologies; Imaging and Chemometrics; Laser-Based and Cavity Ringdown Spectroscopy; Raman, SERS and Security Applications; and Novel 
Spectrometers. In all 38 papers were presented, and we are pleased to be able to bring you 35 of them in these proceedings.

On behalf of our program committee members, we hope that we can count on your participation in a future Next-Generation Spectroscopic Technologies conference.

Mark A. Druy

Richard A. Crocombe 